\title{
Coherent diffraction imaging of a single epitaxial InAs nanowire using a focused x-ray beam
}

\author{
A. Diaz, ${ }^{1,2, *}$ C. Mocuta, ${ }^{1} \dagger$ J. Stangl, ${ }^{2}$ B. Mandl, ${ }^{2}$ C. David, ${ }^{3}$ J. Vila-Comamala, ${ }^{3}$ V. Chamard, ${ }^{4,5}$ T. H. Metzger, ${ }^{1}$ and G. Bauer ${ }^{2}$ \\ ${ }^{1}$ European Synchrotron Radiation Facility, BP 220, F-38043 Grenoble, France \\ ${ }^{2}$ Institute of Semiconductor and Solid State Physics, Johannes Kepler Universität, A-4040 Linz, Austria \\ ${ }^{3}$ Paul Scherrer Institut, CH-5232 Villigen, Switzerland \\ ${ }^{4}$ Aix-Marseille Université, IM2NP, FST Avenue Escadrille Normandie Niemen, F-13397 Marseille Cedex, France \\ ${ }^{5}$ CNRS, IM2NP, FST Avenue Escadrille Normandie Niemen, F-13397 Marseille Cedex, France \\ (Received 4 November 2008; revised manuscript received 9 February 2009; published 27 March 2009)
}

\begin{abstract}
The cross section of an InAs nanowire with a diameter of $150 \mathrm{~nm}$ epitaxially grown on a 111-oriented $\mathrm{InP}$ substrate was characterized using a combination of $\mathrm{x}$-ray scanning microdiffraction and coherent diffraction imaging. Using an x-ray beam focused by a Fresnel zone plate, we were able to scan in real space and hence localize single nanowires on the substrate in the as-grown epitaxial state. For one single nanowire, the threedimensional coherent intensity distribution in reciprocal space was mapped around the (111) InAs reflection. Using phase retrieval algorithms, the cross section of the wire was reconstructed with a spatial resolution of $8 \mathrm{~nm}$ along one direction.
\end{abstract}

DOI: 10.1103/PhysRevB.79.125324

PACS number(s): 61.05.C-, 68.65.-k, 68.37.Yz, 42.30.Rx

\section{INTRODUCTION}

Semiconductor nanowires are very attractive from both fundamental and applicative point of view. They represent one-dimensional electronic systems with typical dimensions in the range of 10-100 nm. Epitaxial growth is possible on a wide range of substrates, rather independent of lattice mismatch and surface orientation. This enables in particular the epitaxial growth of III-V semiconductor nanowires onto Sibased substrates, in which problems with extended defects are avoided due to the small contact area with the substrate. Hence the realization of optically active devices on a Si platform is within reach. This combination of materials can also be used for the fabrication of multijunction solar cells with improved efficiencies. ${ }^{1}$ For all these applications, precise control of growth and resulting nanowire properties is mandatory. Advanced characterization techniques for a minute analysis of nanowires are thus of utmost relevance.

Scanning electron microscopy (SEM) is a widely used technique for the characterization of shape and size of single epitaxial nanostructures, but the crystal structure is not accessible. In combination with SEM, transmission electron microscopy (TEM) can be used for the structure determination and the imaging of defects within the wires. However, in these studies, nanowires need to be taken off the substrate and transferred to another support. X-ray diffraction is an established nondestructive technique for the determination of structure, chemical composition, and strain fields within crystals and for the characterization of nanostructures, ${ }^{2-4}$ being particularly suited for the study of self-assembled epitaxial nanostructures. ${ }^{5}$ Typically a beam of several hundred microns in diameter is used to illuminate many of these structures simultaneously. Reciprocal space maps (RSMs) around Bragg reflections are then compared with calculations based on a model, determining the ensemble-average properties. ${ }^{5-8}$ Although this method is successful, it relies on the choice of a usually simplified model and requires monodisperse systems, while during self-organized growth epitaxial nanowires often show a distribution of all structural pa- rameters. Moreover, the growth of nanowires is often accompanied by other nanostructures during the selfassembly, resulting in an overlap of the (different) diffraction patterns and limiting the significance of such ensembleaveraged measurements. This motivates microdiffraction experiments on individual structures, as recently introduced by Mocuta et al. ${ }^{9}$

Here we present a model-independent method for the study of individual epitaxial nanostructures by means of coherent diffraction imaging (CDI).$^{10}$ This emerging technique allows for the three-dimensional (3D) imaging of nanostructures with a resolution of a few tens of nanometers. ${ }^{11}$ Iterative phase retrieval algorithms ${ }^{12,13}$ are used in order to recover the electron density of a complex-valued object directly from oversampled diffraction patterns. ${ }^{14}$ Strain imaging in a nanocrystal is made possible when CDI is performed in the Bragg geometry. ${ }^{15,16}$ For this purpose, an effective complex-valued electron density is considered, the phase of which is the projection of the displacement field onto the probed Bragg vector. ${ }^{17}$ This imaging technique requires a coherent illumination of the sample and its resolution depends on the extension of the measured coherent diffraction pattern. However, while third generation synchrotron sources deliver highly intense (partially) coherent beams, the coherent flux at the sample position is still too low to address nanostructures within a $10 \mathrm{~nm}$ range resolution. Therefore, in order to achieve a sufficiently strong scattered signal, CDI experiments using highly focused beams have been introduced. ${ }^{18}$ The wave-front curvature produced by a Fresnel zone plate (FZP) is used to improve the convergence of the iterative algorithm. ${ }^{19}$ KirkpatrickBaez focusing mirrors have been employed to measure the coherent x-ray diffraction pattern of a silver nanocrystal at a Bragg reflection. ${ }^{20}$ Moreover, it has been shown that the increase in the coherent flux density at the sample position allows for the reconstruction of the electron-density distribution of a $\mathrm{Au}$ nanostructure with an improved resolution of about $5 \mathrm{~nm} \cdot{ }^{21}$ It has been demonstrated that, under the condition of coherent illumination of the focusing lens, the diffraction-limited spot has a coherence length which is 


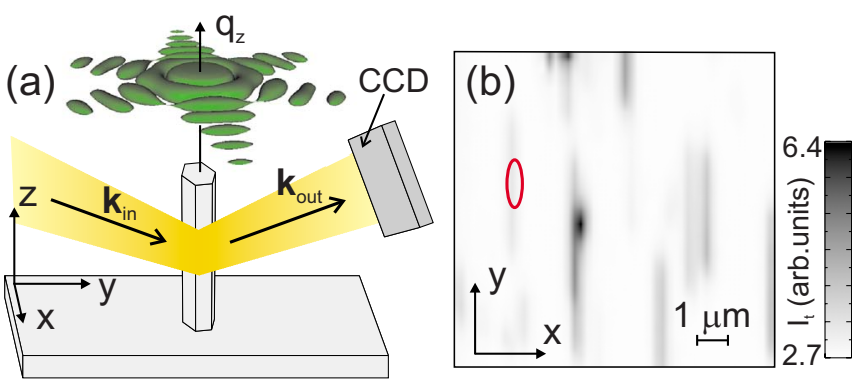

FIG. 1. (Color online) (a) Sketch of the setup. A focused x-ray beam is Bragg diffracted by a single InAs nanowire of $150 \mathrm{~nm}$ diameter. A 3D calculation of the expected scattered intensity close to the InAs (111) reflection is shown (isointensity surface). (b) Distribution of InAs wires on a selected sample area obtained by scanning x-ray diffraction microscopy (linear intensity scale $I_{t}$ ). It shows the projection of several wires along the direction of the incident $\mathrm{x}$-ray beam. The red ellipse indicates the corresponding size of the beam.

larger that the focal spot, enabling coherent diffraction experiments. $^{21}$

In this paper we investigate a single epitaxial InAs nanowire grown on an InP (111) substrate using CDI. While CDI has been previously used for the imaging of individual nanowires detached from the substrate using partially coherent radiation, ${ }^{22}$ here we are able to analyze a single nanowire in its as-grown state due to the efficient focusing of an x-ray beam in the diffraction limit.

\section{EXPERIMENTAL}

As a model sample we used InAs nanowires grown by metal-organic chemical vapor deposition on a 111-oriented InP substrate. ${ }^{23}$ From previous TEM studies ${ }^{24}$ and from finite element method (FEM) calculations (not shown here), it is known that the nanowires, although epitaxially grown, are fully relaxed except for a small region at the wire/substrate interface. These calculations show that significant strain values extend into the wire along the wire axis only for a height roughly corresponding to the wire diameter. The wires have a hexagonal shape, as shown in Fig. 1(a), with an average diameter of $150 \mathrm{~nm}$ and a height of a few microns. The wires grow with a random spatial distribution on the substrate with an average interwire distance of a few microns.

The x-ray diffraction experiment was performed at beamline ID01 at the European Synchrotron Radiation Facility (ESRF) in Grenoble, France. We used an $8 \mathrm{keV}$ x-ray beam from a Si-111 monolithic channel-cut monochromator $\left(\Delta E / E \approx 1.4 \times 10^{-4}\right)$. A FZP made of Au with $200 \mu \mathrm{m} \mathrm{di-}$ ameter and $100 \mathrm{~nm}$ outermost zone width was placed 129 mm upstream of the sample. ${ }^{25}$ A central stop and an order sorting aperture were placed before and after the FZP, respectively, in order to block the higher diffraction orders produced by the lens. In order to produce a diffractionlimited spot size, ${ }^{21}$ an aperture matched to the transversal coherence lengths of the incoming radiation was placed in front of the FZP. ${ }^{26}$ The measured sizes of the focused beam (full width half maximum) were 350 and $400 \mathrm{~nm}$ in the vertical and horizontal directions, respectively. Special care was taken to place the sample exactly into the focal spot. Under these conditions, the illumination of the sample was coherent to a high degree and it was expected that the phase of the wave front was flat in the focus. ${ }^{21}$ A flux density of $\approx 10^{9} \mathrm{ph} / \mathrm{s} \mu \mathrm{m}^{2}$ was obtained. Figure 1(a) shows a sketch of the scattering geometry. The surface of the sample was tilted around the $x$ axis by an angle $\theta$ close to the (111) InAs Bragg reflection $\left(\theta_{B} \approx 12.8^{\circ}\right)$ and the charge coupling device $(\mathrm{CCD})$ detector was placed at an angle $2 \theta_{B}$ at a distance of $904 \mathrm{~mm}$ from the sample. In this geometry, the beam completely illuminated the nanowire in the $x$ and $y$ directions, but only partially in the $z$ direction. The $x$-ray beam illuminates the wire at a position about $1 \mu \mathrm{m}$ above the interface with the substrate, i.e., in a region where the InAs wire adapts its unstrained lattice parameter.

The scattered intensity from a nanocrystal around a Bragg reflection can be calculated from the density and strain distributions within the crystal by means of Fourier transformation. On the top part of Fig. 1(a) we show a calculation of the expected intensity distribution resulting from the illuminated part of the wire close to the (111) InAs Bragg peak. In the particular case in which the crystal is strain-free, the scattered intensity distribution is characteristic for the shape and size of the wire. The sixfold symmetric pattern in the $q_{x} q_{y}$ plane corresponds to the Fourier transformation of a hexagon. The finite region of the illumination of the wire along the $z$ direction is taken into account by a Gaussian modulation of the diffraction pattern in the $q_{z}$ direction.

In order to localize a single nanowire on the substrate, the technique of scanning x-ray microdiffraction (SXMD) was used. ${ }^{9}$ With the geometry shown in Fig. 1(a), the CCD image plane (resulting in a curved surface in reciprocal space) intersects the InAs (111) Bragg position at an angle of $90^{\circ}$ $-\theta_{B}$ with respect to the $q_{z}$ axis. The InP (111) substrate signal lies at much higher $q_{z}$ values and it is not intercepted by the CCD at this incidence angle. Using the InAs (111) reflection as a probe signal, a region of the sample along the $x$ and $y$ directions was scanned in a mesh of $51 \times 51$ points with a step of $200 \mathrm{~nm}$. Figure 1(b) shows the result of the SXMD measurement, consisting of a two-dimensional (2D) plot in which each pixel represents the integrated signal $I_{t}$ of the wire peak on the CCD slice of the corresponding mesh point. We can clearly distinguish the shape of several nanowires projected onto the substrate along the direction of the incoming beam. The scattering intensity is particularly high whenever several nanowires are simultaneously illuminated by the beam due to the rather low Bragg angle.

A single nanowire was selected by moving the sample on a translation stage with nanometer precision to the position indicated by the red ellipse in Fig. 1(b). At this position, the sample was rotated around the Bragg angle $\theta_{B}$ in steps of $0.0075^{\circ}$ (rocking scan) and a CCD image was recorded at each step in order to map the 3D RSM around the (111) InAs reflection. Figure 2(a) shows the measured 3D RSM, in which we observe the sixfold symmetric pattern expected from the Fourier transform of a hexagon around the (111) reflection. Additionally, a streak of intensity along the $q_{z}$ direction arising from the InP substrate crystal truncation rod (CTR) is observed: as the beam goes through the wire, it 

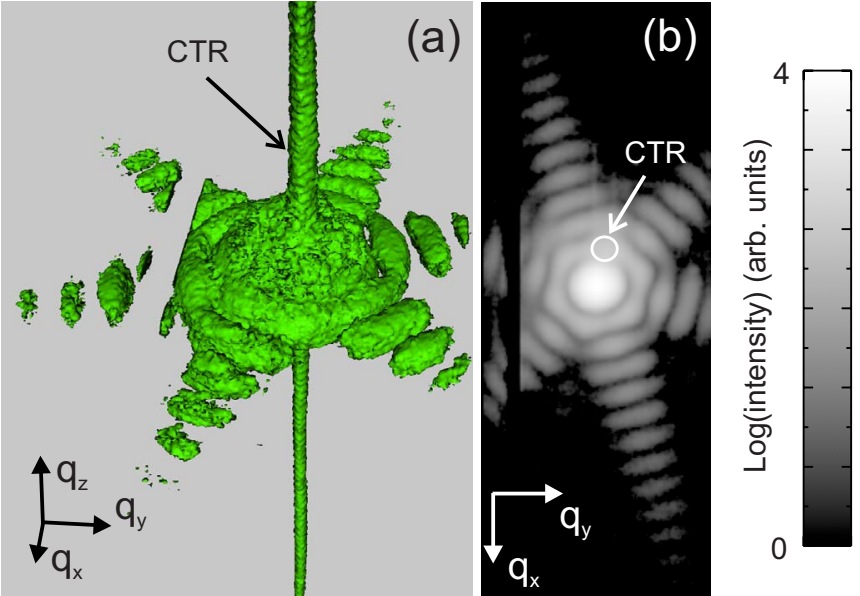

FIG. 2. (Color online) (a) 3D RSM measured around the (111) InAs Bragg reflection. An isointensity surface is shown. The Bragg reflection from the InP substrate appears at higher $q_{z}$ values, out of the plotted data range. (b) 2D cut in the $q_{x} q_{y}$ plane of the RSM shown in (a). The intersection with the CTR is indicated by a circle. Missing data on the left side of the pattern are due to missing frames at scanning points where the CCD intercepts the substrate Bragg reflection.

illuminates a part of the substrate, resulting in the observed CTR. The (111) InP Bragg peak [not shown in the RSM of Fig. 2(a)] is expected at larger $q_{z}$ values. The 3D intensity distribution around the (111) InAs peak is symmetric along the $q_{z}$ direction, indicating a constant lattice spacing in the $z$ direction within the illuminated part of the wire.

\section{DATA ANALYSIS AND DISCUSSION}

Figure 2(b) shows the sum of 11 slices of the measured 3D intensity distribution of Fig. 2(a) in the $q_{x} q_{y}$ plane around the InAs peak. A high-quality diffraction pattern with fringes up to the tenth diffraction order spanning a dynamic range of 4 orders of magnitude is obtained. The InP substrate CTR, indicated by a circle in Fig. 2(b), and the center of the diffraction pattern of the InAs nanowire do not coincide as a consequence of a slight tilting of the nanowire with respect to the surface of the substrate, which could be an indication for defects at the wire/substrate interface.

In order to avoid the CCD saturation close to the (111) InP substrate reflection, the 3D RSM was recorded with shorter exposure times, leading to a degraded signal statistic for patterns measured at these angular positions. Therefore, the frames corresponding to these measurements were removed from the 3D RSM and they appear as a streak of missing data on the left part of the diffraction pattern in Fig. 2(b).

A combination of phase retrieval algorithms, namely, error reduction ${ }^{12}$ and Fienup's hybrid input-output, ${ }^{13}$ was used in order to reconstruct the cross section of the nanowire from the diffraction pattern shown in Fig. 2(b). In the reconstruction, both magnitude and phase of the complex-valued object were let to evolve freely without any constraints, apart from the support: the electron density was set to zero outside a

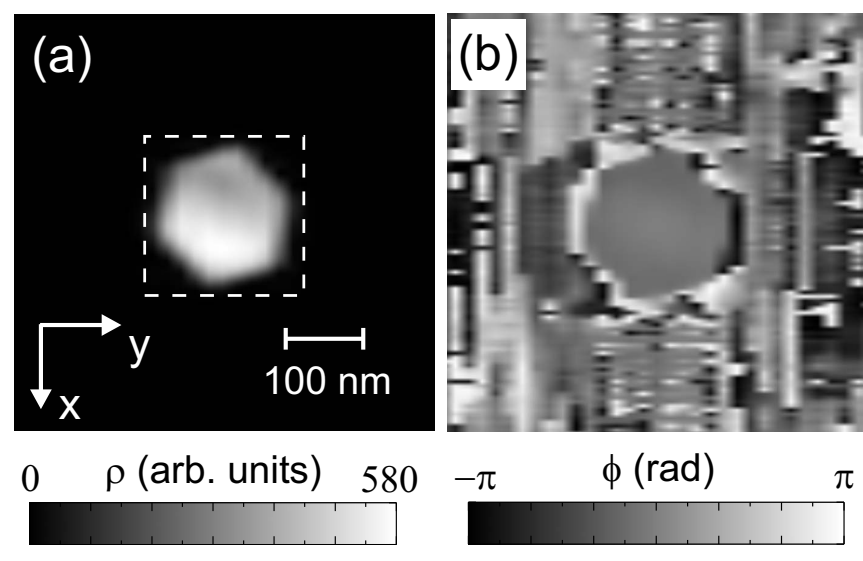

FIG. 3. Retrieved complex-valued electron density-(a) magnitude and (b) phase - of the wire section obtained from the data shown in Fig. 2(b). The dashed square in (a) indicates the support region used in the reconstruction algorithm. Scales in both (a) and (b) are linear.

certain region. At each iteration, the reciprocal space constraints (substitution of magnitudes by the square root of the measured intensities) were applied except for regions of missing data in the diffraction pattern (around the CTR signal and at the streak of missing frames). We performed 100 reconstructions starting with different sets of random phases each time and we averaged the complex-valued solutions. ${ }^{27}$

Figure 3 shows the reconstructed magnitude $\rho$ and phase $\phi$ of the average complex electron density. The dashed line in Fig. 3(a) indicates the support region used in the reconstruction. The magnitude of the retrieved electron density [Fig. 3(a)] has the shape of a hexagon, as expected for the cross section of the InAs wire. Note that, in the reconstruction, we used a support shape with a different symmetry than the one in the wire and a size considerably larger than the wire diameter in order to make sure that the support itself has no influence on the result. We observe that the reconstructed $\rho$ is not homogeneous within the entire cross section of the wire. As explained below, this effect is due to artifacts in the experimental data arising from the fact of measuring an asgrown wire, which introduces regions of missing data in the measured intensity pattern.

The reconstructed phase is shown in Fig. 3(b). We observe that the phase is not well defined at regions of zero $\rho$ values. Within the wire region, the phase shows smooth variations of up to $0.5 \mathrm{rad}$. Since we know that the illuminated part of the wire is strain-free, we attribute these phase variations to artifacts in the experimental data or to a distorted incident wave front onto the sample. The wave-front curvature of the incident beam is a critical issue which should be considered when using CDI for the imaging of strain fields within nanocrystals.

In order to check the influence of the missing data on the phase retrieval result, we preformed a second reconstruction using the data of Fig. 2(b) in which we filled the regions of missing data with the values corresponding to their centersymmetric positions in the diffraction pattern. Note that we do not symmetrize the measured intensity as a whole but use symmetrized data only to replace the missing ones. This is 


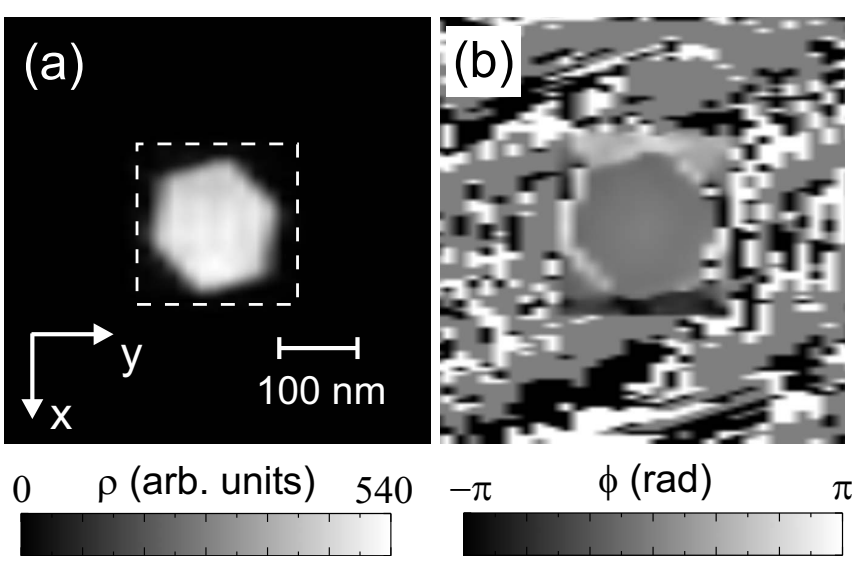

FIG. 4. Retrieved complex-valued electron density-(a) magnitude and (b) phase - of the wire section obtained from the data shown in Fig. 2(b) in which the regions of missing data have been replaced by the center-symmetric values of the diffraction pattern. The dashed square in (a) indicates the support region used in the reconstruction algorithm. Scales in both (a) and (b) are linear.

not strictly valid but a very good approximation having in mind that the illuminated part of the wire is strain-free and, for the energy used, the sample is a weak phase object. The resulting average complex electron density is shown in Fig. 4. In comparison with the reconstruction in Fig. 3(a), the magnitude $\rho$ has the same hexagonal shape and it is now homogeneous, as expected. The reconstructed phase $\phi$ in Fig. 4(b) presents similar phase variations as in Fig. 3(b), suggesting that the missing data affect mostly the magnitude of the electron density.

A natural consequence of iterative phase retrieval algorithms, which make use of fast Fourier transformations, is that the pixel size in the reconstructed image is given by $2 \pi / \Delta, \Delta$ being the extension of the measured diffraction pattern. Since $\Delta$ is different in each direction, the resulting pixel sizes are about $8 \mathrm{~nm}$ along the $x$ direction and $16 \mathrm{~nm}$ along the $y$ direction.

\section{CONCLUSIONS AND OUTLOOK}

We have imaged the cross section of an InAs nanowire of $150 \mathrm{~nm}$ diameter grown on an InP 111-oriented substrate in its as-grown state, without the need of detaching it from the substrate. This was made possible by the combination of SXMD and CDI, together with a focused beam in diffraction-limited conditions, resulting in a high-flux beam with a high degree of coherence over a spot size of 350 $\times 400 \mathrm{~nm}^{2}$. Resolutions of about $8 \mathrm{~nm}$ in the $x$ direction and $16 \mathrm{~nm}$ in the $y$ direction could be achieved.

The presented method offers opportunities for the characterization of more complex epitaxial nanostructures presenting strain fields such as core-shell nanowires. The development of fast readout and large dynamic range detectors and the improvement of the mechanical stability of the setup will allow for the recording of complete data sets and will therefore improve the imaging quality. Concerning the issue of distorted wave fronts, recent developments of CDI such as ptychography $^{28}$ and Fresnel coherent diffractive imaging ${ }^{18,19}$ aim at retrieving both the wave field's amplitude scattered by the sample and the wave field's amplitude of the incident beam at the sample position. However, so far these methods have not been demonstrated for the analysis of strain fields. Finally, smaller beams could be used for the spatially resolved characterization of a nanowire along its axis, especially at the interesting region of the wire/substrate interface, for which no other nondestructive method exists.

\section{ACKNOWLEDGMENTS}

The authors thank the staff of ID01 beamline at ESRF for technical support during the measurements. Financial supports from the FWF Vienna (Grant No. SFB025) and from the EC project SANDiE are gratefully acknowledged.

\footnotetext{
*adiaz@esrf.fr

†Present address: Synchrotron Soleil, L'Orme des Merisiers, St. Aubin, BP 48, 91192 Gif-Sur-Yvette, France.

${ }^{1}$ T. Mårtensson et al., Adv. Mater. (Weinheim, Ger.) 19, 1801 (2007).

${ }^{2}$ V. V. Aristov, A. Y. Nikulin, A. A. Snigirev, and P. Zaumseil, Phys. Status Solidi A 95, 81 (1986).

${ }^{3}$ A. V. Darahanau, A. Y. Nikulin, A. Souvorov, Y. Nishino, B. C. Muddle, and T. Ishikawa, Phys. Lett. A 335, 494 (2005).

${ }^{4}$ M. Tolan, W. Press, F. Brinkop, and J. P. Kotthaus, Phys. Rev. B 51, 2239 (1995).

${ }^{5}$ M. Schmidbauer, M. Hankeand, and R. Köhler, Cryst. Res. Technol. 37, 3 (2002).

${ }^{6}$ A. Malachias, S. Kycia, G. Medeiros-Ribeiro, R. MagalhãesPaniago, T. I. Kamins, and R. S. Williams, Phys. Rev. Lett. 91, 176101 (2003).

${ }^{7}$ A. Hesse, J. Stangl, V. Holý, T. Roch, G. Bauer, O. G. Schmidt, U. Denker, and B. Struth, Phys. Rev. B 66, 085321 (2002).

${ }^{8}$ J. Eymery, F. Rieutord, V. Favre-Nicolin, O. Robach, Y.-M. Ni-
}

quet, L. Fröberg, T. Mårtensson, and L. Samuelson, Nano Lett. 7, 2596 (2007).

${ }^{9}$ C. Mocuta, J. Stangl, K. Mundboth, T. H. Metzger, G. Bauer, I. A. Vartanyants, M. Schmidbauer, and T. Boeck, Phys. Rev. B 77, 245425 (2008).

${ }^{10}$ J. Miao, P. Charalambous, J. Kirz, and D. Sayre, Nature (London) 400, 342 (1999).

${ }^{11}$ J. Miao, T. Ishikawa, B. Johnson, E. H. Anderson, B. Lai, and K. O. Hodgson, Phys. Rev. Lett. 89, 088303 (2002).

${ }^{12}$ R. W. Gerchberg and W. O. Saxton, Optik (Jena) 35, 235 (1972).

${ }^{13}$ J. R. Fienup, Appl. Opt. 21, 2758 (1982).

${ }^{14}$ D. Sayre, Acta Crystallogr. 5, 843 (1952).

${ }^{15}$ I. K. Robinson, I. A. Vartanyants, G. J. Williams, M. A. Pfeifer, and J. A. Pitney, Phys. Rev. Lett. 87, 195505 (2001).

${ }^{16}$ M. A. Pfeifer, G. J. Williams, I. A. Vartanyants, R. Harder, and I. K. Robinson, Nature (London) 442, 63 (2006).

${ }^{17}$ S. Takagi, J. Phys. Soc. Jpn. 26, 1239 (1969).

${ }^{18}$ H. M. Quiney, A. G. Peele, Z. Cai, D. Paterson, and K. A. Nugent, Nat. Phys. 2, 101 (2006) 
${ }^{19}$ G. J. Williams, H. M. Quiney, B. B. Dhal, C. Q. Tran, K. A. Nugent, A. G. Peele, D. Paterson, and M. D. de Jonge, Phys. Rev. Lett. 97, 025506 (2006).

${ }^{20}$ I. K. Robinson, F. Pfeiffer, I. A. Vartanyants, Y. Sun, and Y. Xia, Opt. Express 11, 2329 (2003).

${ }^{21}$ C. G. Schroer, P. Boye, J. M. Feldkamp, J. Patommel, A. Schropp, A. Schwab, S. Stephan, M. Burghammer, S. Schöder, and C. Riekel, Phys. Rev. Lett. 101, 090801 (2008).

${ }^{22}$ V. Favre-Nicolin, J. Eymery, R. Köster, and P. Gentile, Phys. Rev. B (to be published).

${ }^{23}$ B. Mandl, J. Stangl, T. Mårtensson, A. Mikkelsen, J. Eriksson, L. S. Karlsson, G. Bauer, L. Samuelson, and W. Seifert, Nano Lett. 6, 1817 (2006).
${ }^{24}$ M. W. Larsson, J. B. Wagner, M. Wallin, P. Håkansson, L. E. Fröberg, L. Samuelson, and L. R. Wallenberg, Nanotechnology 18, 015504 (2007).

${ }^{25}$ K. Jefimovs, O. Bunk, F. Pfeiffer, D. Grolimund, J. F. van der Veen, and C. David, Microelectron. Eng. 84, 1467 (2007).

${ }^{26}$ The FZP entrance aperture for coherent illumination was defined by slits of opening $80 \times 20 \mu \mathrm{m}^{2}$ (vertical $\times$ horizontal). The slits were off centered with respect to the optical axis of the FZP.

${ }^{27}$ In the averaging process we took into account the two possible types of solutions, namely, the complex-valued density and its center-symmetric complex conjugate.

${ }^{28}$ P. Thibault, M. Dierolf, A. Menzel, O. Bunk, C. David, and F. Pfeiffer, Science 321, 379 (2008). 